\section{Is hyacinth exercise better than pelvic floor exercise alone for urinary incontinence: A randomized trial}

\author{
Maherah Kamarudin, ${ }^{1}$ \\ Jesrine Gek Shan Hong, \\ Yogeeta Gunasagran, ${ }^{1}$ \\ Chua Shiao Chuan, ${ }^{1}$ Noor Adeebah M. \\ Razif, ${ }^{1}$ Su Yen Khong, ${ }^{1}$ Maw Pin Tan ${ }^{2}$ \\ ${ }^{1}$ Department of Obstetrics and \\ Gynecology; '2Department of Medicine, \\ Faculty of Medicine, University of \\ Malaya, Lembah Pantai, Kuala \\ Lumpur, Malaysia
}

\begin{abstract}
Urinary Incontinence (UI) negatively impact women's physical, psychological and health-related quality of life. Pelvic strengthening exercise is the first line management in UI. We hypothesized that Hyacinth exercise is better than Pelvic Floor Exercise (PFE) in strengthening pelvic floor muscles, thus reducing female urinary incontinence. This randomized controlled trial involved 180 women with UI. Participants were taught on either Hyacinth exercise or PFE alone. Improvement in urinary symptoms and pelvic muscle strength were evaluated using a validated female pelvic floor questionnaire and objectively measured at 2 months and 6 months. Significant improvement in urinary symptoms and pelvic muscle strength post treatment at 2 months and 6 months was seen in both groups with $p \leq 0.001$. Although earlier improvement seen in Hyacinth group, it did not reach level of significant when compared, between the two exercises. Mean bladder score (PFE vs Hyacinth arm) $9.13 \pm 6.54$ vs. $8.74 \pm 5.21, \mathrm{p}=0.93$ ( 2 months) and $5.80 \pm 4.15$ vs. $5.79 \pm 4.22, \mathrm{p}=0.92$ (6 months). Although there was no statistical difference between the two groups for our primary and secondary outcomes, Hyacinth group demonstrated earlier improvement in urinary symptoms at 2 months.
\end{abstract}

\section{Introduction}

Almost one in five women experience Urinary Incontinence (UI), affecting their normal daily activities. ${ }^{1}$ Women with incontinence at varying age ${ }^{2,3}$ often suffer in silence and have low self-esteem. Pelvic floor muscle is the main support and provide closure mechanism for urethra to prevent involuntary loss of urine. Muscle tone, maximal strength, speed of contraction, and durability of pelvic floor decline in women with UI. ${ }^{4}$

Management of UI can be complex involving conservative (lifestyle modifications, bladder drills and pelvic floor exercises), medical (anticholinergics, topical estrogen) and surgical measures (mid-urethral slings, colpo-suspension). According to United Kingdom National Institute of Clinical Excellence guidance, a trial of supervised pelvic floor muscle training of at least 3 months, should be offered to women with stress or mixed urinary incontinence before any other intervention. ${ }^{5}$ PFE has also been shown by systematic reviews, ${ }^{6}$ to improve symptoms of SUI and all other types of urinary incontinence. ${ }^{6}$ However, there is no further elaboration on consensus of the best exercise.

'Salat' steps are the repetition of four movements that mimic Muslim prayer steps. It involves common movement and postures that need to be maintained for desired length while focusing on breathing. This promotes balance, wellness, and coordination. Salat emphasizes on increasing strength of the core muscle and gently stretching it to allow gentle muscle contraction. It begins with a standing posture, followed by $90^{\circ}$ bowing, then back to standing before adopting a prostrate position with the forehead, nose, hands, knees, and balls of the toes touching the ground for several seconds. The next steps are sitting directly on the ground, while both legs are folded with the heels touch the gluteal region and the hands are placed on the knees few seconds before rising to standing position. Every movement are followed by a few seconds of resting period before moving to the next steps. One study showed that Salat exercises improved symptoms in women with UI, ${ }^{7}$ whilst another demonstrated that it increases blood flow to the pelvic floor muscles. ${ }^{8}$ This physical movement can treat and rehabilitate the cardiovascular, genitourinary system, at the same time improve impotence in men, quality of sex and genital muscle recovery ${ }^{9}$ which ultimately imply its positive effect to the pelvic floor muscle. Given the diverse benefit of Salat steps, we proposed a study term "Hyacinth" exercise, which combined PFE and Salat steps to see its synergistic effect and hoping to evaluate its effectiveness when compared to PFE alone. "Hyacinth", is a flower which symbolizes sport activities in the Ancient Victorian era.

For the treatment to be effective, proper instruction, motivation, training and feedback are essential, all of which can be further enhanced by using biofeedback devices such as FemiScan. ${ }^{10}$
Correspondence: Maherah Kamarudin, Department of Obstetrics and Gynecology, Faculty of Medicine, University of Malaya, Lembah Pantai, Kuala Lumpur 50603, Malaysia. Tel.: +603 79492059

E-mail: maherah@ummc.edu.my

Key words: Conservative treatment; hyacinth; pelvic floor; Salat exercise; urinary incontinence; pelvic floor exercise

Acknowledgements: We thank our physiotherapist Madam Norlia and Madam Nur Faizah for their involvement in providing knowledge and training to the participants. We thank the women who participated in this trial and their care providers.

Contributions: MK: Project development, data interpretation and manuscript writing; JGSH: Data analysis, manuscript writing and editing; YG: Data collection and initial manuscript writing; CSC: Data collection; NAMR: Data analysis; SYK: Project development and manuscript editing; MPT: Project development. All authors contributed to elements of the study, assert ownership and responsibility for the manuscript.

Conflict of interest: The authors declare no conflict of interest.

Funding: This research was internally funded by the Department of Obstetrics and Gynecology, University of Malaya

Availability of data and materials: All data generated or analyzed during this study are available upon request from corresponding author.

Ethics approval and consent to participate: Clinical trial registry (NCT 02510131), Ethics approval was provided by our institutional review board, the University Malaya Medical Centre (UMMC) Ethics Committee (MECID.NO 20147-390) dated 24 September 2014. The study is conformed with the Helsinki Declaration of 1964, as revised in 2013, concerning human and animal rights. All patients participating in this study signed a written informed consent form for participating in this study.

Informed consent: Written informed consent was obtained from a legally authorized representative(s) for anonymized patient information to be published in this article.

Received for publication: 4 January 2021.

Revision received: 6 August 2021

Accepted for publication: 6 August 2021.

This work is licensed under a Creative Commons Attribution NonCommercial 4.0 License (CC BY-NC 4.0).

(C) Copyright: the Author(s), 2021

Licensee PAGEPress, Italy

Urogynaecologia 2021; 33:266

doi:10.4081/uij.2021.266 
There is no similar study performed before on effectiveness of this exercise on urinary incontinence, we based the hypothesis on its positive effect in the treatment of erectile dysfunction. ${ }^{11}$ In a pilot study ${ }^{7}$ done in our center, improvement in bladder function which was objectively measured with the Female Pelvic Floor Questionnaire (FPFQ) was seen earlier in the Salat plus PFE arm as compared to PFE alone. Based on this, we sought to perform a powered study with a larger cohort and longer follow up period. We hypothesized that Hyacinth exercise (combination of Salat and PFE) are more effective in improving UI symptoms than PFE alone.

\section{Materials and Methods}

\section{Trial design}

The trial was approved by the University Malaya Medical Centre (UMMC) Ethics Committee (Reference number: MECID.NO 20147-39 dated 24 September 2014), and registered at ClinicalTrial.gov website (Registration no. NCT 02510131). The study was conducted in compliance with the Declaration of Helsinki in University Malaya Medical Center. The study was conducted from July 2015 to May 2018 in Gynecology and Geriatric outpatient clinic in University Malaya Medical Centre, a tertiary teaching hospital in Kuala Lumpur.

\section{Participants}

Inclusion criteria were women aged 55 years and above with complaints of urinary incontinence. Exclusion criteria were stage 3 and 4 pelvic organs prolapse, women who are unable or unwilling to provide written informed consent, and those with underlying neurological disorders. Women who previously participated in other therapies to improve pelvic floor muscle were also excluded.

\section{Recruitment and randomization}

Posters about UI problem in women and trial recruitment were placed at the registration counters of the Gynecology and Family Medicine clinic. Interested women were invited to answer a simple questionnaire on UI symptoms. Eligible women were approached, provided with patient information sheet and verbally counseled with regard to trial participation by the co-investigator ( $\mathrm{YG}$ and $\mathrm{CSC}$ ). Written informed consent were obtained from all participants prior to involvement in the study. One-hundred and eighty (180) women fulfilled the inclusion criteria and agreed to participate.

Ninety participants were randomized to either Hyacinth exercise or Pelvic Floor Exercise arm on 1 to 1 ratio by opening the lowest numbered available, sealed and opaque envelope. A total of 180 envelopes sequentially numbered (from 1 to 180) were prepared based on computer generated random sequence in random blocks of 4 by a co-investigator (SYK) who was not involved in the trial recruitment.

\section{Interventions}

At first visit, all relevant demographic data were transcribed onto proformas and participants were asked to fill in self-administered questionnaire using the Australian Pelvic Floor Questionnaire (AFPPQ). ${ }^{12}$ All women then underwent objective measurement of pelvic floor muscle strength using an intravaginal electromyography $\left(\right.$ Femiscan $\left.{ }^{\circledR}\right)$ probe. $^{13}$ These data were recorded and documented as baseline prior to any intervention.

Participants were taught Hyacinth exercise or PFE alone in one-to-one session based on their randomization by a physiotherapist. Participants were provided with an exercise diary to document their daily exercise. Definition of compliance to this study would be, if the participant was able to fulfill more than $>75 \%$ of the prescribed exercise.

Ninety women were trained on pelvic floor muscle exercise, comprising of Slow
Twitch Fiber (STF) and Fast Twitch Fiber (FTF) exercises (Table 1). The STF exercises were targeted at training endurance of the pelvic floor while FTF exercises were aimed to increase strength. Participants were taught STF exercises by contracting their pelvic floor muscles from 1 to $10 \mathrm{sec}$ onds, with a relaxation phase of 10 seconds for 20 cycles. These were tailored according to their ability to contract their muscles. They were also taught 30 repetitive cycles of FTF exercises with 1 second contraction followed by 1 second relaxation. A total of 3 sessions per day was advised.

Another 90 women were taught Hyacinth exercise. This exercise consisted of PFE and Salat steps. PFE as described above added with Salat steps. The Salat steps are the repetition of four movements that mimic the Muslim prayer steps, which starts in a standing posture. Subsequently, the individual bends at the waist placing the hands on the knees while keeping the back straight (ninety degree bowing). The women then returned to the standing position before adopting a prostrate position with the forehead, nose, hands, knees, and balls of the toes touching the ground for several seconds, followed by sitting on the legs for a few seconds before rising to standing. Participants were required to perform Salat steps three times a day in addition of PFE. This equated to an extra 9 minutes and 3 seconds per day (Table 1). Flexibility was added to the routine to enable tailoring to individual ability.

After the first exercise, the participants were seen regularly to review the exercise diaries for compliance, and instructions for their exercise were reinforced. During subsequent visits at 2 and 6 months, they were required to complete the APFQ and measurements of their pelvic muscle strength were recorded. All participants were seen by the same physiotherapist and investigator (YG and CSC) throughout the study period.

Table 1. Pelvic floor exercise and Salat exercises regime.

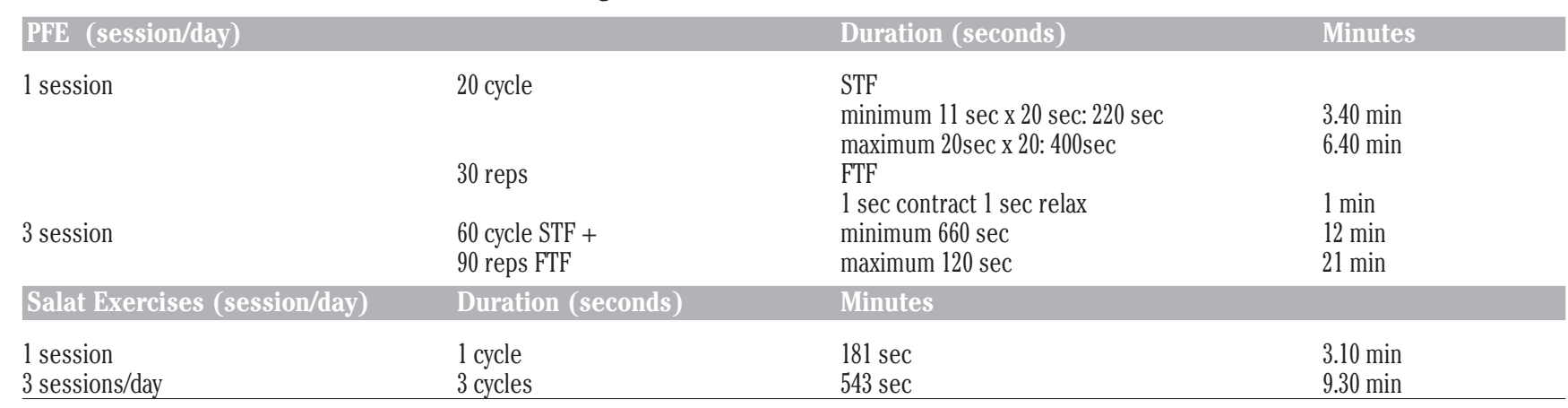




\section{Outcome measures}

Primary outcome was potential improvement in incontinence symptoms by looking at the total score of bladder section from APFQ questionnaire. It consists of 15 questions; the higher score indicates more severe symptoms.

The specified secondary outcomes were objective measurement of pelvic floor muscle strength. Measurements were taken using an intravaginal electromyography $\left(\right.$ Femiscan $^{\circledR)}$ probe. These vaginal probes have electrodes embedded on their surfaces, created a convenient way to measure surface EMG from the pelvic floor muscles. This was more specific than recording electrical potential from the perineum. Five available measurements were recorded namely the Activity of the contractions, the Peak (calculated as the maximum average of time period of $0.3 \mathrm{sec}$ of muscular contraction), the Speed (calculated as muscular activity change from relaxation level to maximum activity level), the Work (calculated as the sum of muscular activity, which corresponds to the area below the activity curve, the total result is the sum of all contraction/relaxation results), and the Relax (calculated as the minimum average level of relaxation between contractions) phase.

For the purpose of this study, the Work was used to measure the strength of the pelvic floor. Participants were asked to do slow twitch fiber muscle exercise and the measurements were recorded for one minute.

\section{Sample size calculation}

The trial was based on superiority hypothesis. Previous pilot ${ }^{7}$ study showed a significant difference when comparing the APFQ score in bladder section for the first and the second visit in the PFE group ( $p=$ 0.046 ). Taking $\alpha=0.05,80 \%$ power, aiming to detect true difference of $\delta=0.46$, and a standard deviation of $1,1: 1$ ratio, and applying student $t$ test,75 experimental subjects were required in each arm. Factoring a $10 \%$ sample increments for Mann-Whitney $U$ test analysis for non-normal distribution and $10 \%$ dropout, the total number of 180 patients were needed.

\section{Data analysis}

Data were entered into a statistical software package SPSS (version 26, SPSS $\odot$ Statistics; IBM Corp., Armonk, NY, USA). Analysis was performed using $t$-test to compare means and distributions for normally distributed continuous data, the MannWhitney $U$ test for non-normally distributed data. All test was two-sided and $\mathrm{P}$ value $<0.05$ was considered significant.

\section{Results}

This study reporting quality was improved using Consolidated Standards of Reporting Trials. Figure 1 depicts the flow of participants into the study. From July
2015 to October 2018, a total of 400 women underwent screening for eligibility. Of the 232 women who were eligible, 180 provided written informed consent and underwent randomization. Ninety were assigned to the PFE arm and the other 90 to the Hyacinth arm. 76 women in PFE arm and 68 in Hyacinth arm were compliant to follow up (6 months).

Table 2 shows the characteristics of the trial participants. These characteristics of the trial participants were similar across trial arms. All of the patients were postmenopausal. There was no significant difference between the groups in terms of Urinary Incontinence (UI) types. More than $70 \%$ of the participants were compliant to dedicated exercise.

Table 3 and 4 report the primary and secondary outcomes dichotomized according to assignment to PFE or Hyacinth trial arms. There were no significant different in bladder score and pelvic muscle strength between the two intervention arms (Table 4), but there was significant improvement in outcome measures between each visit (at recruitment, 2 and 6 months) with $\mathrm{p}<0.001$. Hyacinth arm showed a marginally higher improvement subjectively in bladder score, $12.01 \pm 6.72$ to $8.75 \pm 5.21$ (3.26 difference) $v s$ PFE arm $11.75 \pm 5.98$ to $9.13 \pm 6.54$ (2.62 difference) between recruitment and 2 months visits.

However, at 6 months visit, both arms achieved similar bladder score. Lower bladder score indicates less severe urinary

Table 2. Patient characteristic according to PFE versus Hyacinth exercise.

\begin{tabular}{|c|c|c|c|}
\hline Characteristics & PFE Group ( $n=79)(\%)$ & Hyacinth Group $(n=80)(\%)$ & p \\
\hline Age (years) & $67.33 \pm 6.75$ & $64.31 \pm 5.96$ & 0.217 \\
\hline Ethnicity & & & 0.0034 \\
\hline Malay & $15(19.0)$ & $25(31.3)$ & \\
\hline Chinese & $47(59.5)$ & $46(57.5)$ & \\
\hline Indian & $17(21.5)$ & $7(8.8)$ & \\
\hline Others & 0 & $2(2.5)$ & \\
\hline $\operatorname{BMI}\left(\mathrm{kg} / \mathrm{m}^{2}\right)$ & $24.95 \pm 4.80$ & $25.37 \pm 4.98$ & 0.587 \\
\hline Menopause age (years) & $50.96 \pm 3.67$ & $50.06 \pm 3.88$ & 0.135 \\
\hline Parity & $2.62 \pm 1.59$ & $2.50 \pm 1.48$ & 0.801 \\
\hline Vaginal delivery & $2.18 \pm 1.62$ & $1.90 \pm 1.59$ & 0.625 \\
\hline History of Hysterectomy & $16(20.3)$ & $18(22.5)$ & 0.730 \\
\hline Use of Hormone Replacement Therapy & $16(20.3)$ & $9(11.3)$ & 0.119 \\
\hline Type of Urinary Incontinence (UI) & & & 0.234 \\
\hline Urge UI & $24(30.4)$ & $18(22.5)$ & \\
\hline Stress UI & $25(31.6)$ & $21(26.3)$ & \\
\hline Mixed UI & $30(38.0)$ & $41(51.2)$ & \\
\hline Compliance & & & 0.213 \\
\hline No & $16(20.3)$ & $23(28.8)$ & \\
\hline Yes & $63(79.7)$ & $57(71.3)$ & \\
\hline
\end{tabular}

Data expressed as mean \pm standard deviation or number (\%). Analysis by Student t test for comparison of means for continuous data. P value of significant is $<0.05$ for all analyses. 
symptoms. Hyacinth arm also showed a larger improvement objectively in pelvic muscle strength pre and post treatment at 6 months, $138.31 \pm 129.98$ to $260.77 \pm 274.89$ (122.46 difference) vs $148.37 \pm 244.07$ to $172.58 \pm 147.12$ (24.21 difference) in PFE arm. Higher mean pelvic muscle strength post treatment, although not significant was seen in Hyacinth group with mean of $136.55 \pm 137.13$ vs 201.79 $\pm 282.34, \mathrm{p}=0.085$ (PFE vs. Hyacinth) at 2 months and $172.58 \pm 148.12$ vs $260.77 \pm 274.89, \mathrm{p}=0.200$ at 6 months.

\section{Discussion}

Although there is no statistical difference between the two groups for our primary and secondary outcome, this study shows that Hyacinth group demonstrates earlier improvement in urinary stress incontinence at two months when compared to PFE group. These symptoms continue to improve at 6 months period. When the pelvic muscle activity was objectively measured using the electromyography, both intervention arms showed significant improvement; with the Hyacinth exercise having a marginally higher result, which was similar with previous pilot data. ${ }^{7}$

Our findings concurred with other studies which reported the effectiveness of pelvic floor muscle training on improving incontinence symptoms, and that it should be included as a first-line conservative management for women with UI. ${ }^{4,14}$ A Cochrane review concluded that Pelvic floor muscle exercise can reduce number of leakage episodes and improve symptoms of UI. ${ }^{4}$

There is no consensus on the most effective muscle strengthening exercise or standardized regime for pelvic floor in order to increase bulk and strength. ${ }^{15}$ This study confirms that pelvic floor exercise, with or without addition of Salat steps is an effective conservative measure in reducing urinary leakage. In order to maintain the pelvic floor strength, the recommendation was for patients to perform the exercises in a long-run and consistent manner. One way to achieve this is by introducing exercise

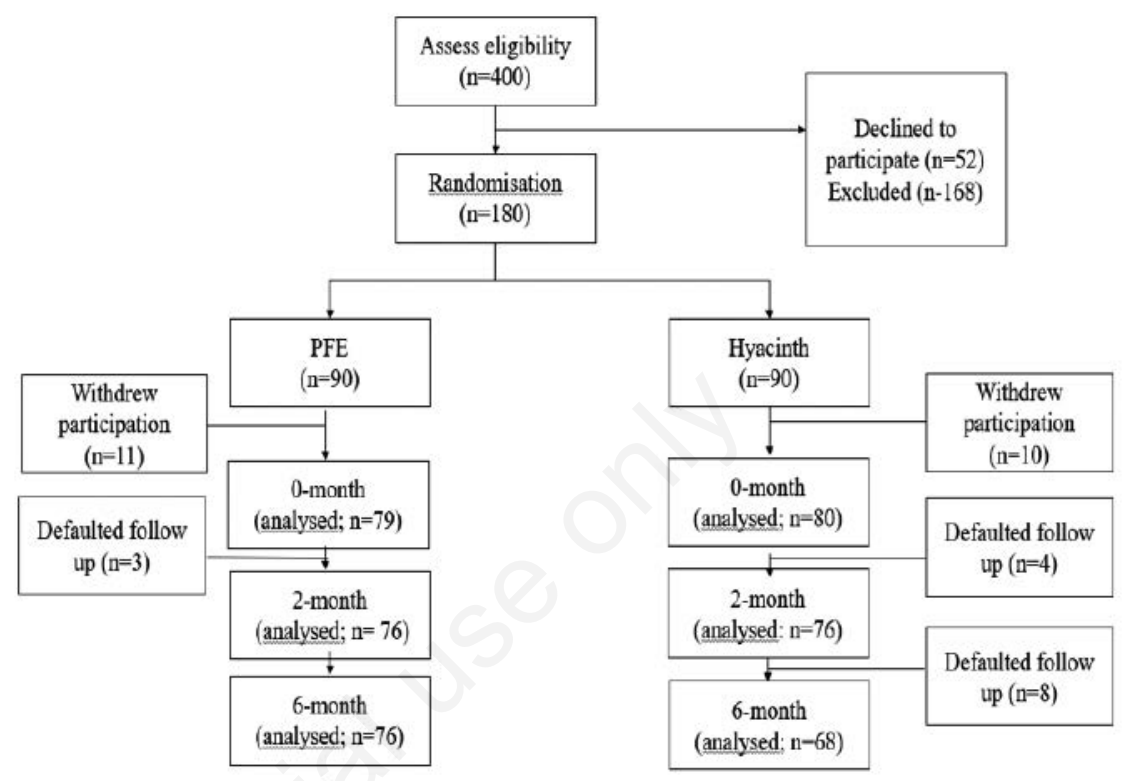

Figure 1. Recruitment flow chart.

Table 3. Comparison of outcome measures between visits.

\begin{tabular}{|c|c|c|c|c|c|c|}
\hline \multirow{2}{*}{$\begin{array}{l}\text { Outcome } \\
\text { Bladder score }\end{array}$} & \multicolumn{2}{|c|}{ PFE } & \multirow[t]{2}{*}{ p } & \multicolumn{2}{|c|}{ Hyacinth } & \multirow[t]{2}{*}{ p } \\
\hline & n & Mean \pm SD & & n & Mean \pm SD & \\
\hline At recruitment & 79 & $11.75 \pm 5.98$ & & 80 & $12.01 \pm 6.72$ & \\
\hline At 2-month & 76 & $9.13 \pm 6.54$ & $<0.001$ & 76 & $8.75 \pm 5.21$ & $<0.001$ \\
\hline At 6-month & 76 & $5.80 \pm 4.15$ & $<0.001$ & 68 & $5.79 \pm 4.22$ & $<0.001$ \\
\hline Pelvic muscle strength & $\mathrm{n}$ & Mean \pm SD & & n & Mean \pm SD & \\
\hline At recruitment & 79 & $148.37 \pm 244.07$ & & 80 & $138.31 \pm 129.98$ & \\
\hline At 2-month & 76 & $136.55 \pm 137.13$ & $<0.001$ & 76 & $201.79 \pm 282.34$ & $<0.001$ \\
\hline At 6-month & 76 & $172.58 \pm 148.12$ & $<0.001$ & 68 & $260.77 \pm 274.89$ & $<0.001$ \\
\hline
\end{tabular}

Data expressed as mean \pm standard deviation. Analysis by Paired $t$ test for comparison of means for continuous data. P value of significant is $<0.05$ for all analyses.

Table 4. Comparison of outcome measures between PFE and Hyacinth arms.

\begin{tabular}{|c|c|c|c|c|c|}
\hline \multirow{2}{*}{$\begin{array}{l}\text { Outcome } \\
\text { Bladder score }\end{array}$} & \multicolumn{2}{|c|}{ PFE } & \multicolumn{2}{|c|}{ Hyacinth } & \multirow[t]{2}{*}{$\mathbf{p}$} \\
\hline & n & Mean \pm SD & n & Mean \pm SD & \\
\hline At At recruitment & 79 & $11.75 \pm 5.98$ & 80 & $12.01 \pm 6.72$ & 0.985 \\
\hline At 2-month & 76 & $9.13 \pm 6.54$ & 76 & $8.75 \pm 5.21$ & 0.937 \\
\hline At 6-month & 76 & $5.80 \pm 4.15$ & 68 & $5.79 \pm 4.22$ & 0.915 \\
\hline Pelvic muscle strength & n & MeantSD & $\mathbf{n}$ & MeantSD & \\
\hline At recruitment & 79 & $148.37 \pm 244.07$ & 80 & $138.31 \pm 129.98$ & 0.682 \\
\hline At 2-month & 76 & $136.55 \pm 137.13$ & 76 & $201.79 \pm 282.34$ & 0.085 \\
\hline At 6-month & 76 & $172.58 \pm 148.12$ & 68 & $260.77 \pm 274.89$ & 0.200 \\
\hline
\end{tabular}

Data expressed as mean \pm standard deviation. Analysis by Independant $t$ test for comparison of means for continuous data. P value of significant is $<0.05$ for all analyses. 
that patient is comfortable and familiar with. ${ }^{16}$

The result of our study showed significant impact by 2 months after exercise was initiated. Although the regime and character of the pelvic muscle contraction is different, a study by Donahoe-Fillmore et al., showed improvement can only be seen by 12 weeks after initiation of the exercise. ${ }^{17}$ Repetitive movement in Hyacinth exercise not only coordinate the core muscle, but also strengthen and improve flexibility of muscles and ligaments. ${ }^{18}$ Our study showed significant improvement in incontinence symptoms and stronger pelvic muscle strength when women were followed up till 6 months duration. However, it did not prove the assumption that Hyacinth exercise is better than doing pelvic exercises alone.

This study has shown that Hyacinth can significantly provide faster improvement objectively through increase in muscle strength and marginally better improvement in bladder score even at two-month. There was also no negative impact to the pelvic floor seen with exercise. Women can see this as an option, to be added to their routine PFE and those already familiar with the 'Salat steps' who adopt these postures during their prayers should be encouraged to perform Hyacinth to improve adherence, whilst further improve their quality of life. This study has shown previous multiple positive benefits of 'Salat steps' on overall health, improvement in balancing, cognitive, lower back pain, improve blood supply to pelvic as well as lower limb. These steps are regarded as a gentle muscle contraction and painless thus suitable across ages. It is also easy to be replicated by anyone.

\section{Strengths and limitations}

This is by far the largest cohort study with longer follow-up period reviewing the effect of traditional pelvic floor exercise versus Salat steps incorporated into the PFE on urinary incontinence. Previous pilot study involved only 30 patients and shorted follow-up period. The participants were seen by the same investigator and physiotherapist throughout the study period to minimize bias. The strength of this study included the in-depth assessment of the urinary symptoms subjectively using validated APFQ and objectively using a Femiscan ${ }^{\circledR}$.

Several identifiable weaknesses of this study that affect the outcome, thus differ its results from the initial pilot study done were identified. These include, patient compliance to exercise and follow up. At the end of 6 months follow up, a total of 12 patients dropped out from Hyacinth group. Only 3 dropped out seen from PFE group. Some patients from the Hyacinth group who dropped out from the study complained of having dizziness during prostration. This may be contributed to their age. Some found the Hyacinth exercise to be time consuming and joint pain hindered further exercises. More frequent reviews with monthly measurement of PFM may improve their compliance to the exercise regime and at the same time encouragement can be given to boost their motivation.

Hyacinth exercise does cause improvement in urinary symptoms and has the ability to improve pelvic floor muscle strength. To the best of our knowledge, there is no one standardized PFE, which has been shown to be most effective in improving urinary incontinence. Women can be taught various exercises to tailor to their preferences (length of program, frequency and duration).

Future studies should further evaluate which exercise protocol will have maximal impact on women with UI, follow up strategies and frequency of monitoring using additional objective tools that can boost patient motivation in order to facilitate better compliance.

\section{Conclusions}

Hyacinth exercise improves urinary incontinence symptoms earlier by two months, though not statistically different compared with conventional pelvic floor exercise. Combination of Salat and PFE may improve adherence, promote consistency and maintain pelvic floor muscle strength. However, future research is needed to ascertain its cost-effectiveness as compared to medical vs surgical option.

\section{References}

1. Sims J, Browning C, LundgrenLindquist B, Kendig H. Urinary incontinence in a community sample of older adults: prevalence and impact on quality of life. Disabil Rehabil 2011;33:1389-98.

2. Swanson JG, Kaczorowski J, Skelly J, Finkelstein M. Urinary incontinence: common problem among women over 45. Can Fam Physician 2005;51:84-5.

3. Sandvik H. Female urinary incontinence Studies of epidemiology and management in general practice. Acta Obstetricia et Gynecologica Scandinavica 1996;75:952-4.

4. Dumoulin C, Cacciari LP, Hay-Smith EJC. Pelvic floor muscle training versus no treatment, or inactive control treatments, for urinary incontinence in women. Cochrane Database Syst Rev 2018;10:Cd005654.

5. Smith A, Bevan D, Douglas HR, James DJB. Management of urinary incontinence in women: summary of updated NICE guidance. BMJ 2013;347:f5170.

6. Bø K. Pelvic floor muscle training in treatment of female stress urinary incontinence, pelvic organ prolapse and sexual dysfunction. World J Urol 2012;30:437-43.

7. Khong SY, Lilywaty I, Akmar Z, et al. Alternative slow and moderate exercises for management of pelvic floor muscles dysfunction. Neurourol Urodynam 2016;35:S90-S1.

8. Ibrahim F, Sian TC, Shanggar K, Razack AH. Muslim prayer movements as an alternative therapy in the treatment of erectile dysfunction: a preliminary study. J Phys Ther Sci 2013;25: 1087-91.

9. Alabdulwahab SS, Kachanathu SJ, Oluseye K. Physical activity associated with prayer regimes improves standing dynamic balance of healthy people. J Phys Ther Sci 2013;25:1565-8.

10. Segal S, Morse A, Sangal P, et al. Efficacy of FemiScan pelvic floor therapy for the treatment of urinary incontinence. Female Pelvic Med Reconstr Surg 2016;22:433-7.

11. Ibrahim FB, Sian TC, Shanggar K, Razack AHA. Muslim Prayer Movements as an Alternative Therapy in the Treatment of Erectile Dysfunction: A Preliminary Study. Journal of Physical Therapy Science. 2013;25:1087 - 91 .

12. Baessler K, O'Neill SM, Maher CF, Battistutta D. A validated self-administered female pelvic floor questionnaire. Int Urogynecol J 2010;21:163-72.

13. Auchincloss CC, McLean L. The reliability of surface EMG recorded from the pelvic floor muscles. J Neurosci Methods 2009;182:85-96.

14. Mercier J, Morin M, Zaki D, et al. Pelvic floor muscle training as a treatment for genitourinary syndrome of menopause: A single-arm feasibility study. Maturitas 2019;125:57-62.

15. Braekken IH, Majida M, Engh ME, Bø K. Can pelvic floor muscle training reverse pelvic organ prolapse and reduce prolapse symptoms? An assessor-blinded, randomized, controlled trial. Am J Obstet Gynecol 2010;203:170.e1-7.

16. Cavkaytar S, Kokanali MK, Topcu HO, et al. Effect of home-based Kegel exercises on quality of life in women with 
stress and mixed urinary incontinence. $\mathrm{J}$ Obstet Gynaecol 2015;35:407-10.

17. Donahoe-Fillmore B, Chorny W, Brahler CJ, et al. A comparison of two pelvic floor muscle training programs in females with stress urinary incontinence: a pilot study. J Appl Res 2011;11:73-83.

18. Kamran G. Physical benefits of (Salah) prayer-strengthen the faith and fitness. J
Nov Physiother Rehabil 2018;2:43-53.

19. Nazish N, Kalra N. Muslim prayer-a new form of physical activity: a narrative review. Int $\mathrm{J}$ Health Sci Res 2018;8:337-44 